\title{
Kinematic and thermal characteristics of Lüders and Portevin-Le Châtelier bands in a medium Mn transformation-induced plasticity steel
}

\author{
X.G. Wang ${ }^{\mathrm{a}, \mathrm{b}}$, L. Wang ${ }^{\mathrm{c}}$, M.X. Huang ${ }^{\mathrm{b}, *}$

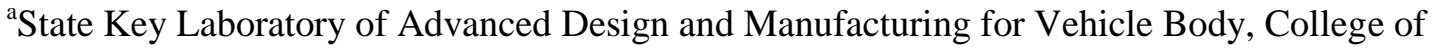 \\ Mechanical and Vehicle Engineering, Hunan University, 410082 Changsha, China \\ ${ }^{\mathrm{b}}$ Department of Mechanical Engineering, The University of Hong Kong, Pokfulam Road, Hong \\ Kong, China \\ ${ }^{\mathrm{c}}$ State Key Lab of Development and Application Technology of Automotive Steels, Baosteel \\ Research Institute, 201900 Shanghai, China \\ * Corresponding author. Tel.: +852 28597906; fax: +852 28585415 \\ E-mail: mxhuang@hku.hk
}

\section{Abstract:}

This paper focuses on experimental investigations on the kinematic and thermal aspects of the propagative plastic instabilities, namely Lüders band and Portevin-Le Châtelier (PLC) band, observed in a medium Mn transformation-induced plasticity (TRIP) steel. The full-field strain and heat source measurements are used concurrently to investigate in-situ the propagative bands. The kinematic analysis provides the real-time strain field evolution allowing the observation and quantification of the band nucleation and propagation. The local Lüders strain is demonstrated both space and time independent, and its magnitude is equivalent to the macroscopic Lüders strain. The spatio-temporal independent characteristic is also verified on the local strain in the PLC bands. Concerning the thermal field analysis, the heat sources are estimated and used to obtain the geometrical and dynamic parameters of the propagating bands, including the band width, orientation, propagation velocity, and appearance moment. Then the estimated heat sources are applied to establish an experimental energy balance during the plastic deformation. The quantitative energy analysis reveals that the strain-induced martensitic transformation takes place in the Lüders band but barely in the PLC bands. The ex-situ X-ray diffraction measurement on the phase content and surface hardness testing of the material confirm well this determination. 
Keywords: Mn TRIP steel; Plastic deformation; Deformation band; Thermal effects; Martensitic transformation

\section{Introduction}

Transformation-induced plasticity (TRIP) steels possess high tensile strength as well as excellent formability so that they are widely used for the body-in-white of automobiles. The mechanical properties of TRIP steels depend largely on the stability and volume fraction of the retained austenite. During the plastic deformation, the retained austenite in TRIP steels transforms to martensite, resulting in enhanced work hardening rate and improved formability [1]. Recently, a new type of TRIP steels, alloyed with a medium Mn content (5 to 8 wt.\%) and possessing a high volume fraction of retained austenite (20 to $40 \%$ ), has been developed as the "third generation" Advanced High Strength Steels (AHSS) grades for automotive applications[2-4]. These new TRIP steels show exceptional tensile properties with a high tensile strength of 1000-1500 MPa and total elongation of 31-44\% [5]. However, it was also remarked in the literature $[6,7]$ that some plastic instability phenomena, such as the Lüders banding, may occur in the medium Mn TRIP steels during tensile test. It is known that the plastic instability is usually associated with localized and propagative bands on the material surface that may bring unexpected surface roughening effect and lead to the weakening of material-structural stability and even premature failure in some most unfavorable cases. Hence, it has been a topical subject to investigate the plastic instability problem as addressed by numerous studies [8-10].

The plastic instability occurred in metal deformation has two typical forms embodied in propagative bands, which are Lüders bands and Portevin-Le Châtelier (PLC) bands. The Lüders bands refer to the regions of localized strain starting from the yield point drop and corresponding to a predominant stress plateau stage. Lüders bands are commonly caused by static strain aging (SSA). The PLC bands represent a variety of irregular inhomogeneous deformations usually manifested as stress serrations (jerky flow) or strain jumps in the tensile stress-strain curve. PLC bands are normally characterized by the dynamic strain aging (DSA) effect. A classical interpretation of these phenomena is based on the interactions between solute 
atoms (such as carbon atoms) and mobile dislocations, originally proposed by Cottrell and Bilby [11] and further developed by Cottrell [12], Van den Beukel [13] and McCormick [14], et al. In the SSA process, it is commonly postulated that the mobile dislocations can be pinned temporally by the interstitial carbon atoms cluster, called Cottrell atmosphere. During the pinning time (or waiting time) the flow stress increases continuously until the dislocations enable to break away from the pinning carbon atoms, and it leads to, consequently, an easier glide of dislocations on the slip plane and then the required stress for the slip movement reduces to lowered levels than before. If the pinning and depinning process manifests in a repetitive manner, it will lead to a DSA effect with the jerky flow in the mechanical response. Another well-known theory attributing the stress drop to multiplication of dislocations was proposed by Johnston and Gilman [15] and Hahn [16], which also allowed demonstrating the up-down-up stress-strain response even in the complex loading conditions [17].

In general, the Lüders band takes place immediately after the onset of plastic deformation at the upper yield stress $\sigma_{U}$, followed by a stress plateau (lower yield stress $\sigma_{L}$ ) characterized by the yield point elongation (YPE), or called macroscopic Lüders strain $\left(\varepsilon_{L}\right)$. The values of these Lüders parameters depend on a variety of factors, e.g., alloy content, grain size, strain rate, and temperature. Comparing to the Lüders bands, the occurrence of the PLC bands is much more irregular and can be embodied in varied forms. Normally the PLC bands can be classified into three types, namely, type A, type B and type C. Type A bands are continuous propagating bands initiated at a certain location (often at a specimen extremity) and moving along the specimen. Type B bands are hopping bands propagating in the axial direction of the specimen. Type $\mathrm{C}$ bands appear as static bands that manifest in a spatially non-correlated manner. It was found that the different types of PLC bands were associated with distinct serration patterns in the stressstrain response [18] and [19]. Similar to Lüders bands, the occurrence and specific form of the PLC bands depended on a set of loading conditions and internal parameters [20].

The spatio-temporal characteristics of the propagative bands were investigated by using acoustic emission technique [21], laser scanning extensometry [22], digital speckle pattern interferometry [23]. More recently, the application of digital image correlation (DIC) method 
[24] allows visualization of the band nucleation and propagation process in real time and at varied length scales from microscale to macroscale. Moreover, it can provide useful physical quantities of displacement and strain for characterizing the localized deformation phenomena in a two-dimensional space. The enriched information that DIC offers promotes greater understanding of the heterogeneous deformation process and dynamic strain aging effect $[8,25]$. Another emerging full-field measurement technique, infrared thermography (IRT), has also been successfully applied to study the strain localization in metallic materials [26]. Thanks to the full-field assessment of temperature and heat sources by IRT, thermal aspects of the propagative bands under tensile load were investigated $[27,28]$. These advanced experimental means provide efficient ways to obtain detailed knowledge on the kinematic and thermal characteristics of the plastic instabilities.

Regarding the medium Mn TRIP steel concerned in this work, our previous study [7] shows that it may manifest both Lüders effect and PLC effect under the tensile load, leading to complex plastic instabilities during deformation. It is important to understand its localized deformation mechanism and to have a thorough knowledge about the band formation and propagation behavior, which are, however, rarely reported in the literature. In the present study, we attempt to employ both DIC and IRT methods for an experimental investigation of the kinematic and thermal characteristics of the propagative plastic instabilities. The combination of the strain and temperature measurements could provide a complete observation of the deformation localization and development process, and obtain enriched information of the spatio-temporal characteristics of the propagative bands. Furthermore, the heat sources derived from the temperature fields can be used for building an experimental energy balance during the plastic deformation. Thus, potentially it provides a new way to discern the deformation mechanism from an energetic viewpoint. The objective of this paper is to study the kinematicthermal aspects of plastic instability behaviors in the medium Mn TRIP steel with wellestablished analysis approaches, and then to understand these effects from the local thermomechanical response. 


\section{Materials and methods}

\subsection{Material and experiments}

The studied material is a recently developed medium Mn TRIP steel. The material has a chemical composition of Fe-7Mn-0.14C-0.23Si (wt.\%). It was cold rolled, followed by an intercritical annealing and air cooling to room temperature, leading to two dominant phase contents: ferrite and austenite [7]. The phase map of the steel is observed by electron backscatter diffraction (EBSD) and is shown in Fig. 1.

The tensile specimen was flat dog-bone specimen with a $32 \mathrm{~mm}$ of the gauge length, $6 \mathrm{~mm}$ width and $1.4 \mathrm{~mm}$ thickness. Tensile tests were conducted at room temperature and at a constant cross-head velocity of $0.02 \mathrm{~mm} / \mathrm{s}$, corresponding to a macroscopic axial strain rate about $6.2 \times$ $10^{-4} \mathrm{~s}^{-1}$. The test was suspended until the failure of the specimen. Different experimental means were employed during and after the tensile test, including the in-situ full-field measurements by DIC and IRT and ex-situ measurements using X-ray diffraction (XRD) and microhardness test.

The kinematic field measurement was carried out using a visible-light camera Elphel NC353L, equipped with a Navitar Zoom 7000 lens. This combination allowed providing a high image resolution of $2592 \times 1944$ pixels and a physical resolution of $0.021 \times 0.021 \mathrm{~mm}$ per pixel in the field of view of $54.4 \mathrm{~mm} \times 40.8 \mathrm{~mm}$. The sampling frequency of the camera was 15 frames per second, which was considered "rapid" comparing to a relatively low loading velocity imposed, allowing, therefore, a "real-time" recording of the deformation history of specimen surface. Before the tensile test, the specimen surface was sprayed with a speckled paint using an airbrush, which provided a black-white mixed random pattern in the captured images of the specimen surface. It served as the carrier of deformation information. Then the DIC computation can be performed on these images in order to evaluate the displacement and strain fields of specimen surface during deformation.

The thermal field measurement was realized by employing a high performance IR camera FLIR X6530 sc. The camera had a very fine thermal sensitivity, with a noise level typically less than $0.02{ }^{\circ} \mathrm{C}$ at room temperature. It provided an image resolution of $512 \times 640$ pixels and 
physical resolution of $0.165 \times 0.165 \mathrm{~mm}$ per pixel in the field of view of $105.6 \mathrm{~mm} \times 105.6 \mathrm{~mm}$. A high sampling frequency of $50 \mathrm{~Hz}$ was adopted. And the specimen surface was deposited by a layer of matt black paint to provide a high and uniform emissivity (about 0.93 ). The IR camera was calibrated before the mechanical test that permitted an accurate temperature measurement.

In this study, the ex-situ measurements were utilized mainly as the verification means on the deformation mechanisms as revealed by the in-situ kinematic-thermal measurements. The employed ex-situ measurements included XRD and hardness test.

\subsection{Methods of image processing}

Visible images of the specimen surface acquired during deformation were processed by DIC method for the assessment of displacement and strain fields. The DIC software adopted in this work was elastix [29], an open source toolbox for image registration. B-spline functions were employed as the shape function in the DIC algorithm for better modeling a heterogeneous deformation process. Each B-spline function was formulated in a correlation window of $48 \times 48$ pixels, applying fine calculation steps down to $1 \times 1$ pixel. And the nonlinear interpolation strategy was adopted, which allowed an ultimate correlation precision on the sub-pixel level. For the detailed procedure of the DIC method, the reader is referred to Ref [29].

The DIC computation provided the axial and transverse displacement fields $\left(U_{x}, U_{y}\right)$, and by differential, four in-plane strain components $\left(\varepsilon_{x x}, \varepsilon_{y y}, \varepsilon_{x y}, \varepsilon_{y x}\right)$. In this work, the axial strain $\left(\varepsilon_{y y}\right)$ was used as the main element in the result analysis concerning the uniaxial tensile test. Thus, the axial strain $\varepsilon_{y y}(x, y)$ for each pixel $(x, y)$ in the zone of interest (ZOI) of specimen surface is now accessible from the DIC computation.

Several strain measures are necessary to be introduced for the strain field analysis. First, the overall average axial strain in the ZOI, or so-called the 0D axial strain, can be defined by

$$
\overline{\bar{\varepsilon}}_{y y}=\frac{1}{M N} \sum_{j=1}^{N} \sum_{i=1}^{M} \varepsilon_{y y}(i, j)
$$

where $\overline{\bar{\varepsilon}}_{y y}$ represents the overall average axial strain, $M$ and $N$ are the total numbers of pixels along the transverse and axial directions, respectively. 
The $1 \mathrm{D}$ axial strain can be defined as the average strain along the axial direction $y$, i.e.,

$$
\bar{\varepsilon}_{y y}(y)=\frac{1}{M} \sum_{i=1}^{M} \varepsilon_{y y}(i, j)
$$

where $\bar{\varepsilon}_{y y}(y)$ represents the average axial strain for a given axial position $y$.

The above strain definitions are the conventional cumulative strains by taking the initial undeformed state as the reference. Here it is also interesting to introduce an incremental expression of strain as a measure of the current deformation state. The incremental strain can be defined by

$$
\Delta \varepsilon^{i}=\varepsilon^{i}-\varepsilon^{i-1} \quad(i=1,2, \ldots, n)
$$

where $i$ represents the reference number of the acquired image, and $\Delta \varepsilon^{i}$ stands for the incremental strain for the $i$ th image.

Adopting the incremental strain definition, both the axial strains in OD and 1D can be expressed in the incremental form, as $\Delta \overline{\bar{\varepsilon}}_{y y}$ and $\Delta \bar{\varepsilon}_{y y}$, respectively. One advantage of adopting the incremental strains is to identify the "currently" deforming areas in the strain field, and then to further link these features to the work hardening effect or other material behaviors [30].

Concerning the IRT measurement, the temperature variation of specimen surface during deformation is the direct output of a calibrated IR camera. Nevertheless, comparing to temperature, the heat source is considered more intrinsic to the deformation mechanisms, and is used more often in the thermomechanical analysis. A methodology dealing with the passage from the measured temperature fields to heat sources was established in the framework of continuum thermomechanics and was well-documented in the work of Chrysochoos and Louche [31]. Due to the limitation of space, here we shall only present the most important results and the pertinent models adopted in this work.

The study [31] shows that a compact form of energy balance in an irreversible deformation process can be obtained under a set of hypotheses (e.g., small perturbation hypothesis, negligible convective terms), which can be expressed by

$$
\rho C \frac{\partial \theta}{\partial t}-k \Delta \theta=s
$$


where $\rho$ is the mass density, $C$ the specific heat, $k$ the isotropic thermal conductivity, $t$ the current time, $\theta=T-T_{0}$ the temperature difference between the absolute temperature $T$ of specimen surface and the ambient temperature $T_{0}$, while $s$ stands for the overall heat source due to energy dissipation and other coupling effects, e.g., thermoelasticity.

The heat diffusion term is taken into consideration by the Laplacian operator in the above equation. The heat sources are then evaluated from discrete derivation operators, in time and space, applied to the temperature field $\theta(x, y, t)$. The heat diffusion problem can be solved in two-dimensional space or more simply in one-dimensional space, leading to 2D model and 1D model, respectively.

The 2D heat diffusion model takes advantage of all the acquired thermal data in a twodimensional space. It takes the form

$$
\rho C\left(\frac{\partial \theta}{\partial t}+\frac{\theta}{\tau_{t h}^{1 D}}\right)-k\left(\frac{\partial^{2} \theta}{\partial x^{2}}+\frac{\partial^{2} \theta}{\partial y^{2}}\right)=s
$$

where the time constant $\tau_{t h}^{1 D}$ characterizes the heat loss through the specimen surfaces.

The studies $[27,32]$ show that the application of the $2 \mathrm{D}$ model requires a good signal-tonoise ratio and preliminary low pass filtering operations. For the cases of a less satisfactory signal-to-noise ratio and diffuse thermal data, a 1D heat diffusion model was proposed. It assumes that the averaged heat sources located along the axial direction allow representing the thermal manifestations occurred on the whole specimen surface. And it takes the form

$$
\rho C\left(\frac{\partial \bar{\theta}}{\partial t}+\frac{\bar{\theta}}{\tau_{t h}^{2 D}}\right)-k\left(\frac{\partial^{2} \bar{\theta}}{\partial y^{2}}\right)=\bar{s}
$$

where $\bar{\theta}$ stands for the temperature averaged through the transverse direction in the thermal images and the time constant $\tau_{t h}^{2 D}$ characterizes the heat loss at the cross-section boundaries.

In addition to the $1 \mathrm{D}$ and $2 \mathrm{D}$ models, an even simpler solution, so-called $0 \mathrm{D}$ model, can be considered. Its underlying hypothesis is that the heat source distribution, before localization onset, stays uniform at any time within the specimen gauge part. In such a case, the spectral solution of the heat equation can be approximated by using the first eigenfunction of the 
Laplacian operator. Thus, the heat equation can be simplified as

$$
\rho C\left(\frac{\partial \overline{\bar{\theta}}}{\partial t}+\frac{\overline{\bar{\theta}}}{\tau_{e q}}\right) \approx \overline{\bar{s}}
$$

where $\overline{\bar{\theta}}$ represents the average temperature variation in the specimen gauge part, with $\tau_{e q}$ characterizing the overall heat loss.

The $0 \mathrm{D}$ model can provide an effective way to estimate the overall heat source. In the present study, the established thermal diffusion models from $0 \mathrm{D}$ to $2 \mathrm{D}$ were all employed by taking their unique advantages according to the specific applications.

\section{Results and Discussions}

\subsection{Macroscopic mechanical behavior}

The macroscopic engineering stress-strain curve of the specimen in the tensile test, obtained using an extensometer, is illustrated in Fig. 2. The material manifests an elasto-plastic behavior, in which a typical yield point drop (from point A to B) appears shortly after the elastic regime and followed by a stress plateau (from point $\mathrm{B}$ to $\mathrm{C}$ ). The latter phenomenon is normally attributed to the Lüders banding effect. After that, the material is hardened with continuing straining, and the stress enhancement demonstrates a serrated pattern. This phenomenon is typically associated with the PLC banding effect. A close-up observation on the jerky stress is shown within Fig. 2. It exhibits that the jerky flow is composed of a series of serrations with irregular forms and unequal amplitudes. It is in good agreement with the description of the Type A PLC bands according to [18]. For ease of presentation, the whole deformation process of material is divided into three stages. They are stage I - elastic deformation stage, stage II stress plateau stage (or Lüders banding stage), and stage III - work hardening stage (or PLC banding stage), as marked in Fig. 2.

Some tensile properties of the test material can be derived from the macroscopic stressstrain curve and are listed in Table 1. It includes the upper yield stress $\sigma_{U}$, lower yield stress $\sigma_{L}$, ultimate tensile strength (UTS) $\sigma_{U T S}$, YPE $\varepsilon_{y}$, Lüders strain $\varepsilon_{L}$ and elongation to failure $\varepsilon_{f}$. It can be remarked that the medium Mn TRIP steel exhibits an excellent combination of strength and 
ductility, with a $\sigma_{U T S}$ of $1012 \mathrm{MPa}$ and $\varepsilon_{f}$ of 0.423 . It is also noticeable that the evaluated Lüders strain is as high as 0.095 . This value is apparently higher than the values typically observed in ferritic steels [33] or other TRIP steels [6] and [34]. It corresponds to an abnormally long stress plateau in the stress-strain curve, occupying a section about $22.45 \%$ of the total elongation. The rest and predominant plastic deformation is possessed by the work hardening regime associated with the PLC bands. Hence, one may consider that the whole irreversible deformation process of the medium Mn TRIP steel is accompanied with plastic instability phenomena. It is, therefore, of great importance to gain sufficient knowledge about the spatio-temporal characteristics of the propagative bands. In this work, the primary experimental means are the full-field strain and temperature measurements and heat source estimation. By these methods, the spatio-temporal features of the propagative bands are investigated in both kinematic and thermal aspects.

\subsection{Kinematic aspects}

The studies $[8,26]$ show that the mapping of $1 \mathrm{D}$ strain evolution allows presenting a full picture of the localized deformation behavior, including its both spatial and temporal features. Thus, the evolution of the 1D axial strain $\bar{\varepsilon}_{y y}$ during the whole deformation process is plotted, as shown in Fig. 3a.

The three deformation stages I-III are labeled in the displayed strain map, distinguished by the dotted line. It can be noted that some strain localizations are present in stages II and III after the initial stage of homogeneous elastic deformation until about $40 \mathrm{~s}$. At the onset of plasticity in stage II, a Lüders band characterized by high strain localization appears at the lower end of the specimen and propagates continuously towards the other end. In this regime, two areas with the shape of the right-angled triangle can be distinguished along the trace of band propagation. The first area on the top refers to the regions unexperienced by Lüders band where the elastic regime is still dominant. It is named after the non-Lüders area. The second area on the bottom refers to the regions experienced by Lüders band, so-called the Lüders area. It can be remarked that both areas demonstrate homogeneous strain distribution but with pronounced difference in absolute strain values. After stage II dominated by a single Lüders band, one can observe that 
repetitive PLC bands appear and propagate with varied velocities (embodied in different slopes in Fig. 3a) and propagation lengths in stage III. All these bands exhibit a continuous propagating feature and can, therefore, be determined as the Type A PLC band [18]. It is in agreement with the previous determination based on the macroscopic stress-strain variation pattern.

As strain is a cumulative measure, Fig. 3a may not be the most appropriate way for the visualization of the band propagation process. If the strain is expressed in the incremental form, it should be able to better exhibit the position of the strain-localized band front and the kinematic features of the propagative bands. To this end, the evolution of the $1 \mathrm{D}$ axial incremental strain $\Delta \bar{\varepsilon}_{y y}$ with an interval of 3 images (or $0.2 \mathrm{~s}$ ) is illustrated in Fig. $3 \mathrm{~b}$.

Fig. $3 \mathrm{~b}$ shows that the propagating band fronts are much more distinct in the incremental strain expression comparing to Fig. 3a. As a matter of fact, the incremental strain represents the "current" deformation. Thus, one can remark that the "current" deformation primarily occurs at the band front. This effect is more evident in the Lüders banding stage, where the incremental strains in the regions other than the band front are approaching to zero. In the PLC banding stage, the phenomenon is similar but more complicated. First, the PLC bands manifest more localized feature and are possessed of higher propagation velocities than that of the Lüders band. The band propagation length is also varied between the different bands. Moreover, the concurrence of more than one propagating band is somehow possible. Here it is worthy to note that the absolute error and uncertainty level in the DIC computation increases with the deformation level. And this effect may become relatively important when the strain is expressed in the incremental form. It can partially explain the non band-related patterns appeared in stage III. Moreover, the noises are also present in stage I. It is mainly due to the effect of an unsatisfactory signal-to-noise ratio under the small deformation condition.

A unique advantage of the full-field measurement is the access of the local strain. It provides, therefore, a way to evaluate the local strain evolution within the propagating bands. It will be the focus of the following analysis.

It is known that the Lüders band normally nucleates at one end of the specimen due to 
stress concentration and then propagates towards the other end. A detailed description on the band nucleation process is, however, generally absent in the literature. It should be attributed to the fact that the period of the band nucleation is often short during which the strain evolution within the band is not readily accessible by the conventional experimental means. In this study, the DIC method enables a real-time recording of the surface deformation in a two dimensional field of view. Thus the nucleation process of the Lüders band can be investigated in a proper manner. Here the local Lüders strain refers to the local strain within the Lüders area, which is different to the definition of the macroscopic Lüders strain $\varepsilon_{L}$ based on the stress-strain curve.

The Lüders band starts to nucleate at the moment of the stress drop at the termination of the homogenous elastic deformation. The development of the Lüders band can be depicted by Fig. $4 \mathrm{a}$, in which a succession of $2 \mathrm{D}$ strain $\left(\varepsilon_{y y}\right)$ fields at the selected moments during the band nucleation is displayed. It shows that the Lüders band starts to nucleate at the lower end of the specimen and develops progressively during the deformation. The Lüders band exhibits a stationary feature in the nucleation process. The local Lüders strain increases continuously until the complete development of the Lüders band. Fig. $4 \mathrm{~b}$ shows the variation of the mean Lüders strain (averaged in the Lüders band) as a function of time. It can be noted that the mean Lüders strain increases quasi linearly with time during the nucleation process. It takes about $12 \mathrm{~s}$ in total for the band formation. The local Lüders strain increases from an initial value of elastic strain about 0.0045 to a high level near 0.09 at the termination of band nucleation. Thus the local strain rate is about $7 \times 10^{-3} \mathrm{~s}^{-1}$. After that the Lüders band becomes nonstationary and starts to propagate along the specimen.

Concerning the Lüders band propagation stage, Fig. 5a exhibits a succession of selected axial strain $\left(\varepsilon_{y y}\right)$ fields during the whole propagation process, i.e., from the onset of band propagation at the moment $t_{B}$ to its termination at the moment $t_{C}$. Two noticeable observations can be remarked from the strain field evolution. First, the strain is homogenous in the Lüders area, and meanwhile, the strain is equally homogenous but shows considerably lower values in the non-Lüders area. In fact, this observation has already been noted in the 1D strain field analysis (Fig. 3a). Second, the size of the Lüders area increases continuously due to band 
propagation, but the local strain in the Lüders area remains constant during the deformation. Analogously, the strain in the non-Lüders area does not demonstrate any variation in stage II. In the combination of these two effects, it leads to the apparent observation of two "uniform" right-angled triangles in both $1 \mathrm{D}$ and $2 \mathrm{D}$ strain field evolution. For a better and quantitative illustration, the strain evolution of three representative points on the specimen surface is shown in Fig. 5b. The three selected points (A, B and C) are located at the bottom, middle, and top of the gauge section, respectively, as labeled in Fig. 5a. And the six selected moments are also corresponding to six strain fields illustrated in Fig. 5a.

Fig. 5b shows clearly that the strain for a point undeformed by Lüders band (in the nonLüders area) remains equal to the initial elastic strain at the yield point (i.e., the YPE), about 0.0045 in absolute values. Once the point is deformed by Lüders band (entering into the Lüders area), it demonstrates an abrupt strain increment to an elevated level, and then it shows quasi constant during the remaining Lüders band deformation occurred in other regions. It implies that local Lüders strain is neither space-dependent nor time-dependent, and can therefore be characterized by a single value under the ideal conditions. Here the local Lüders strain is denoted by $\varepsilon^{L B}$. At the particular moment $t_{C}$ when the gauge section of specimen surface is $100 \%$ occupied by Lüders area, the macroscopic Lüders strain $\varepsilon_{L}$ equals to the average strain in the Lüders area. Then it equals to the local Lüders strain $\varepsilon^{L B}$ as well. The following relationship can therefore be established:

$$
\varepsilon_{L}=\varepsilon^{L B}=\varepsilon^{L B}(x, y, t) \quad\left(t_{B} \leq t \leq t_{C}\right)
$$

The value of the local Lüders strain $\varepsilon^{L B}$, derived from the experimental strain fields, is about 0.089 in average with little spatio-temporal variation. The value of $\varepsilon_{L}$ is 0.095 as provided by the macroscopic stress-strain curve in which the strain was measured by extensometer. These two values are rather comparable with a difference only about $6 \%$. This analysis demonstrates that the plastic deformation in stage II is solely contributed by the localized Lüders strain, and the global strain increases in the way by extending the Lüders area. A technical remark is that the DIC measurement provides a possibility to determine the level of Lüders strain at the very 
beginning of band propagation.

The 1D strain field analysis has revealed that a number of PLC bands emerge and propagate in succession during the work hardening stage. Each of the PLC bands is possessed of different propagation velocity and operating range. Further analysis shows that the nucleation of the PLC band is much faster than that of the Lüders band. Thus the detailed process of the PLC band nucleation is not available in the present study owing to a relatively low sampling frequency. The analysis is then focused on the band propagation process. It is interesting to quantify the local strain within the PLC band (so-called local PLC strain) and to verify its spatial and temporal dependency.

An example analysis is focused on the first PLC band. Fig. 5c illustrates a succession of selected axial strain $\left(\varepsilon_{y y}\right)$ fields during the band propagation. The PLC band nucleates at the upper end of the specimen and propagates towards the other end. It terminates, however, not at the extremity of the specimen but at a certain position in the middle, as shown by the 6th strain field in Fig. 5c. To verify the spatio-temporal dependency of the local PLC strain, three points (A, B and C) at different positions on the specimen surface are selected for the analysis. Their strain evolutions during the band propagation are shown in Fig. 5d. The point A experiences no PLC deformation, thus its strain value keeps quasi constant during the whole process, which is approximately 0.092 . In contrast, the point $\mathrm{C}$ is experienced by the PLC deformation at the very beginning and is invariably located in the PLC area. Its strain value also shows a constant value of 0.102 with small fluctuations during the band propagation (in other regions). Thus it implies that the local PLC strain is not temporal-dependent. Concerning the point B located in the middle of the specimen, a strain burst is observed when the point is deformed by PLC band. And then it is on the same strain level with the point $\mathrm{C}$, and its strain value becomes stabilized during the remaining PLC banding course. It suggests that the local PLC strain is also spatialdependent. In other words, the strain is supposed to be uniform in the PLC area. The same phenomenon is also observed in other PLC bands. Hence, it can be concluded that the local strain in a given PLC band is neither space-dependent nor time-dependent during the band propagation. The analysis also suggests that the plastic deformation in stage III is mainly 
contributed by the localized PLC strain, and the global strain increases in a similar way with the Lüders band.

In addition to the local strain evaluation, the 2D strain mapping enables the visualization of the band propagation process. Thus, some geometrical parameters (e.g., band width, band orientation) and dynamic parameters (e.g., propagation velocity, appearance frequency) of the PLC bands are possible to be obtained. Regarding the geometrical characterization, the strain field can only identify the band front but not the full geometry of the band as the strain is a cumulative measure of deformation. Nevertheless, this problem can be solved by resorting to the thermal field measurement. Our analysis demonstrates that the estimated heat sources from temperature fields permit the visualization of the propagative band, and thus can be used for the geometrical characterization. Concerning the dynamic parameters, both of the two methods DIC and IRT are, in principle, capable of evaluating the characteristic parameters such as the band propagation velocity. The difference relies mainly on the temporal resolution of the imaging system. In the present study, both the geometrical and dynamic parameter assessments of the PLC bands are based on the heat source analysis since it is more comprehensive for the relevant characterization.

\subsection{Thermal aspects}

Thermal measurement provides an alternative viewpoint to investigate the irreversible deformation process of the medium Mn TRIP steel. Fig. 6 shows the evolution of average

temperature increment $\overline{\bar{\theta}}$ versus strain during the tensile test (the blue curve). In the initial elastic stage, the temperature decreases with increasing stress due to thermoelasticity. Once the plasticity commences with Lüders band formation, the temperature begins to grow rapidly at an almost constant rate during the Lüders banding stage. When it enters the work hardening stage associated with PLC banding effect, the apparent temperature manifests fluctuation with alternating increase and decrease evolution trend, but the net temperature increment is very limited. The overall heat source $\overline{\bar{s}}$ was calculated and its variation versus strain is also displayed in Fig. 6 (the red curve). It can be remarked that the evolution trend of the heat source 
is, on the whole, similar to that of the temperature since they all characterize a global heat development. The only difference is that the fluctuation of the heat source shows more intense. Here it is considered as essential to analyze the local thermal manifestation in connection with the propagative bands for a better understanding of the plastic instability. Hence, the 1D model was applied as the kinematic field analysis. Fig. 7a shows the evolution of the 1D temperature increment $\bar{\theta}$ along the loading direction during the tensile test.

The temperature evolution in Fig. $7 \mathrm{a}$ demonstrates the same phenomenon with the 1D strain evolution (Fig. 3a), though they are expressed in different physical quantities. In stage I, the temperature is uniformly distributed with negative values during the elastic deformation. Then with the onset of plasticity in stage II, a Lüders band nucleates at the lower end of the specimen and propagates towards the other end. It leads to important temperature increment at the propagating band front and, consequently, heat diffusion across the whole specimen surface. After that the emergence and propagation of repetitive PLC bands dominate the rest plastic deformation, pertaining to the work hardening effect in stage III. It can be noted that the temperature shows relatively stabilized in this stage from the first PLC band to the last one, though it is not homogenously distributed along the loading direction. To better interpret the thermal manifestation and its intrinsic correlation with the propagative bands, the estimated heat sources were analyzed. Fig. $7 \mathrm{~b}$ shows the evolution of the 1D heat source $\bar{s}$ during the tensile test. It is corresponding to the temperature evolution in Fig. 7a.

The heat source evolution in Fig. 8 describes well the band propagation process, similar to the effect of the incremental strain expression shown in Fig. 3b. Moreover, it provides solid evidence that the heat is primarily generated at the band front. The propagation paths of the Lüders band and PLC bands can now be more readily determined. Similar to the kinematic observation, the PLC bands differ in propagation length and velocity. The most pronounced PLC bands propagate from the upper end of the specimen to the lower end, and the ones with much less propagation length operate inversely. It can be also remarked that all the involved bands, no matter Lüders band or PLC bands, show heat source intensity on a comparable level. 
In Fig. 8, the evolution of temperature $(\theta)$ and heat source $(s)$ versus strain for a given pixel on the upper half of the specimen surface is illustrated. The crests in temperature or heat source correspond to the moments when the Lüders band or PLC bands pass the given point. The variations in heat source at these moments, after taking into account of the heat diffusion effect, exhibit much sharper than the corresponding temperature variations. Hence, the analysis demonstrates that the heat source may be a more sensitive and intrinsic parameter for characterizing the propagative plastic instability. On one hand, it can be used for the band characterization (both geometrical and dynamic parameters), in particular for the PLC bands. On the other hand, an experimental energy balance analysis on the propagative bands is possible to be performed thanks to the heat estimation.

In this work, the geometrical and dynamic characterization of the plastic instability is primarily focused on the PLC bands. In the 1D heat source evolution shown in Fig. 7b, eight principal PLC bands, propagating from the upper end of the specimen to the lower end, can be observed. They represent the main activity of the PLC banding and are therefore selected for the analysis. Concerning the eight principal PLC bands, their representative heat source fields with the PLC bands located at approximately the same position are illustrated in Fig. 9a. It shows that the eight PLC bands are possessed of more or less the same band width, except for the last two bands where the heat source is not well-shaped due to a bad signal-to-noise ratio probably caused by a low band propagation velocity at the last phase of deformation. The axial profiles of the eight representative heat source fields along the midline of the specimen are shown in Fig. 9b. It demonstrates that the band widths of the eight PLC bands are rather comparable. Their estimated values are in the range from $1.60 \mathrm{~mm}$ to $1.80 \mathrm{~mm}$, with an average of $1.75 \mathrm{~mm}$. This estimate is very close to the estimated width of the Lüders band that is equal to $1.82 \mathrm{~mm}$. Similar to the band width, the band orientation (or inclination angle) also shows rather stable for the repetitive PLC bands. The estimated inclination angles with respect to the loading axis are around $54^{\circ}$ with a small deviation of $\pm 3^{\circ}$, as measured from the heat source fields in Fig. 9a.

It can be remarked from the 1D heat source evolution (Fig. 7b) that the velocity of the PLC bands exhibits a general descending trend with increasing deformation. Along with this 
descending trend in velocity, the propagation length of the bands also becomes shorter, and the interval between two successive bands becomes longer. The estimated velocities of the eight principal PLC bands $\left(V_{P L C}\right)$ are plotted in Fig. 10a as a function of axial strain.

Except the first band showing an irregular low velocity, the estimated velocities of the concerned PLC bands demonstrate a quasi linear decreasing trend with strain varying from the initial $1.76 \mathrm{~mm} / \mathrm{s}$ to $0.62 \mathrm{~mm} / \mathrm{s}$ at the end. These estimates are considerably higher than the evaluated velocity of the Lüders band which is about $0.30 \mathrm{~mm} / \mathrm{s}$. The PLC band velocity $V_{P L C}$ can be well related to axial strain through a linear regression as shown in Fig. 10a. The linear regression was realized using the method of least squares, formulated as below

$$
V_{P L C}=-9.78 \varepsilon+2.77
$$

It is also noted in Fig. 7b that the appearance frequency of the PLC bands becomes lower with increasing strain. In other words, the time interval of the successive bands increases with axial strain. Here a parameter $\Delta T_{P L C}$ is defined as the interval between the end of one PLC band and the start of the next PLC band. In Fig. 10b, $\Delta T_{P L C}$ is plotted as a function of axial strain for the eight principal PLC bands. It shows that the interval $\Delta T_{P L C}$ increases exponentially with the axial strain that varies from $22 \mathrm{~s}$ to $62 \mathrm{~s}$. Their relationship can be approximated using a power function

$$
\Delta T_{P L C}=8065 \varepsilon^{3.2}+17.77
$$

The evolution trend of the current PLC bands in the medium Mn TRIP steel is similar to the observations in $[27,28]$ for, respectively, a 4 wt.\% $\mathrm{Mg}$-Al alloy and a 4 wt.\% $\mathrm{Cu}-\mathrm{Al}$ alloy. The empirical formulas of Eqs. (9) and (10) may be useful for predicting the dynamic behaviors of the PLC bands, though an accurate prediction seems still difficult in view of the complex spatio-temporal nature of the propagative PLC bands.

From a thermomechanical point of view, the plastic deformation is an irreversible dissipation process. In general, most of the plastic work will be converted into heat, and the remaining will be stored within the material, so-called stored energy (or latent energy). Thus, an energy balance can be built in a plastic deformation with the following form 


$$
W_{p}=Q_{p}+E_{s}
$$

where $W_{p}$ represents the plastic work, $Q_{p}$ the heat dissipation and $E_{s}$ the stored energy.

Conventionally, a linear relationship between the plastic work and heat dissipation is assumed through a well-known parameter $\beta$, representing the heat conversion ratio. According to the calorimetric experiments conducted by Rusinek and Klepaczko [34], the parameter $\beta$ in TRIP steels varies within the limits from 0.82 to 1 . In this analysis, a most commonly assumed value 0.9 was adopted as the estimate of $\beta$. Thus,

$$
\beta=\frac{Q_{p}}{W_{p}}=0.9
$$

Then, the dissipated energy can be evaluated through

$$
Q_{p}=\beta W_{p}=0.9 \sigma: \varepsilon^{p}
$$

where $\varepsilon^{p}$ represents the plastic strain.

Here it is important to note that the energy term $Q_{p}$ represents the heat release exclusively associated with the plastic work. Possibly it could be only a portion of the total heat release considering that there may be additional energy input except the plastic work, for instance, the exothermal martensitic transformation. The verification is straightforward in this work, as the total heat dissipation $Q_{\text {total }}$ can be effectively evaluated by the direct calorimetric analysis. By integrating the heat source for a given period, the $Q_{\text {total }}$ can be solved through

$$
Q_{\text {total }}=\int \overline{\bar{s}} d t=\int \rho C\left(\frac{\partial \theta}{\partial t}+\frac{\theta}{\tau_{e q}}\right) d t
$$

Both the energy terms $Q_{p}$ and $Q_{\text {total }}$ refer to the energy estimated on the global scale, i.e., the specimen scale. The $Q_{p}$ can be obtained using the macroscopic stress-strain curve via Eq. (13), and the $Q_{\text {total }}$ using the estimated heat source $\overline{\bar{s}}$ by 0D model via Eq. (14). In this analysis, the two energy terms were calculated for stage II and stage III, respectively. The results are shown in Table 2. Here a parameter $\delta$ is introduced to represent the relative difference between the two quantities, defined as

$$
\delta=\frac{Q_{\text {total }}-Q_{p}}{Q_{p}} \times 100 \%
$$


Table 2 shows that the estimated $Q_{\text {total }}$ is higher than $Q_{p}$ by approximately $56.4 \%$ in stage II, and their values show rather comparable in the subsequent stage III with a difference only about 2.6\%. Hence, it provides reliable evidence that an additional energy input other than the plastic work must have participated in the Lüders banding process. And the most likely energy source is the exothermal martensitic transformation. Concerning the investigated medium Mn TRIP steel, a high volume fraction of retained austenite is existed within the material as the metastable phase, which can be possibly transformed into martensite under tension within the highly strainlocalized Lüders band.

Concerning the verification of martensitic transformation, a direct way is to examine the volume fraction change of austenitic phase in the process of Lüders deformation. It can be realized by the direct measurement of phase content using the XRD technique. Fig. 11a exhibits the evolution of the volume fraction of austenite versus strain at the selected moments in a tensile test. The six selected measurement points are located in the three deformation stages. For the two points in stage II, the measurements were carried out in the Lüders area in order to examine the effect of Lüders deformation. In Fig. 11a, one can remark that little phase transformation takes place in stage I. The variation of austenite volume fraction is only about $2 \%$ from the initial undeformed state to the upper yield point. Then a sharp decrease in austenite volume fraction occurs in stage II, from about $29 \%$ to $9 \%$. It means that about two thirds of the retained austenite has been transformed into martensite in this stage. So stage II accounts for the major portion of martensitic transformation, which is also responsible for the pronounced heat dissipation at this stage. After that, a gradual decrease of austenite volume fraction can still be noted in stage III, from about $9 \%$ to eventually $0 \%$ before fracture. This observation is consistent with the fact that the evaluated $Q_{\text {total }}$ is still a little higher than $Q_{p}$ at this stage. It implies that the martensitic transformation is invariably responsible for the extra heat dissipation in addition to the plastic strain energy.

Since the martensitic transformation in Lüders band is now well confirmed, it could be expected that the hardness of the specimen surface that experienced Lüders band should be increased considerably as martensite is much harder than the retained austenite. A verification 
test using the hardness measurement was therefore conducted on a specimen surface partially experienced by Lüders deformation. The Vickers hardness (HV) measurements were carried out on both the Lüders area and non-Lüders area, and the results of the selected points along the loading axis are plotted in Fig. 11b. A distinct difference in HV values can be noted between the Lüders area and non-Lüders area. The HV of the former is about 321 in average and of the latter only about 268 . The enhancement of $\mathrm{HV}$ is about $20 \%$ as induced by the Lüders deformation.

\section{Conclusions}

The tensile behavior of a $7 \mathrm{wt} . \%$ Mn TRIP steel was investigated in this paper. The studied material exhibited a desirable combination of strength and ductility, with an ultimate tensile strength of $1012 \mathrm{MPa}$ and elongation to failure of $42.3 \%$. Nevertheless, noticeable propagative instabilities were produced during the plastic deformation, including both Lüders and PLC banding effects. They led to an abnormally long stress plateau and a subsequent work hardening stage. Being different from the many of current studies, the principal experimental means adopted in this work was the in-situ strain and temperature field measurement. The kinematic and thermal characteristics of Lüders and PLC bands were characterized and quantified.

Concerning the kinematic aspect of the plastic instability, the analysis was focused on the evolution of the local strain within the propagative bands. It showed that the Lüders band nucleated at one end of the specimen due to stress concentration, in which the local strain grows quasi linearly at a rate about $7 \times 10^{-3} \mathrm{~s}^{-1}$ in a time period of $12 \mathrm{~s}$. After the formation, the band propagated towards the other specimen end at a constant velocity. The local Lüders strain was proved both space-independent and time-independent, and its quantity was equal to the macroscopic Lüders strain. This spatio-temporal independency of local strain was also verified in the PLC bands. The research demonstrated that the local strain development played an important role in the stretching mechanism of the present TRIP steel. The global strain was primarily enhanced by extending the areas occupied by Lüders and PLC deformation.

Regarding the thermal field analysis, the heat source estimation method first showed its utility in characterizing the geometrical and dynamic features of the propagative bands. The 
band characterization parameters, such as the band width, orientation, propagation velocity, were estimated thanks to the full-field visualization by the in-situ measurement. The analysis showed that the successive PLC bands manifested a variation tendency of decreasing propagation velocity and increasing interval of appearance, which can be well related to macroscopic strain using a linear or power equation. The fitted empirical equations could be useful for predicting the PLC banding behavior under suitable conditions. Another more interesting application of the heat source estimation was the establishment of an experimental energy balance in the plastic deformation. It gave a reliable demonstration of the strain-induced martensitic transformation occurred in Lüders band that was responsible for the additional heat contribution other than the mechanical energy input during deformation. This determination was verified by both phase content measurement using XRD and surface hardness testing. The energy balance analysis also demonstrated that there were no or very limited martensitic transformation occurred in the PLC bands.

\section{Acknowledgments}

M.X. Huang acknowledges the support from the Steel Joint Funds of the National Natural

Science Foundation of China (Grant No. U1560204), National Science Foundation of China (Grants No. 51301148), Research Grants Council of Hong Kong (Grants No. HKU719712E, HKU712713E) and Small Project Funding of HKU (Grant No. 201409176053).

\section{References}

[1]P.J. Jacques. Transformation-induced plasticity for high strength formable steels, Current Opinion in Solid State and Materials Science 8 (2004) 259-265.

[2]P.J. Gibbs, E. De Moor, M.J. Merwin, B. Clausen, J.G. Speer, D.K. Matlock. Austenite Stability Effects on Tensile Behavior of Manganese-Enriched-Austenite TransformationInduced Plasticity Steel, Metallurgical and Materials Transactions A 42 (2011) 3691-3702.

[3]S. Lee, S.-J. Lee, B.C. De Cooman. Austenite stability of ultrafine-grained transformation-induced plasticity steel with Mn partitioning, Scripta Materialia 65 (2011) 225228.

[4]B.B. He, M.X. Huang, Z.Y. Liang, A.H.W. Ngan, H.W. Luo, J. Shi, W.Q. Cao, H. Dong. Nanoindentation investigation on the mechanical stability of individual austenite grains in a medium-Mn transformation-induced plasticity steel, Scripta Materialia 69 (2013) 215-218.

[5]J. Shi, X. Sun, M. Wang, W. Hui, H. Dong, W. Cao. Enhanced work-hardening behavior and mechanical properties in ultrafine-grained steels with large-fractioned metastable austenite, Scripta Materialia 63 (2010) 815-818. 
[6]H. Luo, H. Dong, M. Huang. Effect of intercritical annealing on the Lüders strains of medium Mn transformation-induced plasticity steels, Materials \& Design 83 (2015) 42-48.

[7]X. Wang, L. Wang, M. Huang. In-situ evaluation of Lüders band associated with martensitic transformation in a medium Mn transformation-induced plasticity steel, Materials Science and Engineering: A 674 (2016) 59-63.

[8]J. Min, L.G. Hector, J. Lin, J.T. Carter, A.K. Sachdev. Spatio-temporal characteristics of propagative plastic instabilities in a rare earth containing magnesium alloy, International Journal of Plasticity 57 (2014) 52-76.

[9]H. Aboulfadl, J. Deges, P. Choi, D. Raabe. Dynamic strain aging studied at the atomic scale, Acta Materialia 86 (2015) 34-42.

[10] R. Sarmah, G. Ananthakrishna. Correlation between band propagation property and the nature of serrations in the Portevin-Le Chatelier effect, Acta Materialia 91 (2015) 192201.

[11] A.H. Cottrell, B. Bilby. Dislocation theory of yielding and strain ageing of iron, Proceedings of the Physical Society. Section A 62 (1949) 49.

[12] A. Cottrell. LXXXVI. A note on the Portevin-Le Chatelier effect, The London, Edinburgh, and Dublin Philosophical Magazine and Journal of Science 44 (1953) 829-832.

[13] A. Van den Beukel. Theory of the effect of dynamic strain aging on mechanical properties, Physica status solidi (a) 30 (1975) 197-206.

[14] P. McCormick. Theory of flow localisation due to dynamic strain ageing, Acta Metallurgica 36 (1988) 3061-3067.

[15] W. Johnston, J.J. Gilman. Dislocation velocities, dislocation densities, and plastic flow in lithium fluoride crystals, Journal of Applied Physics 30 (1959) 129-144.

[16] G.T. Hahn. A model for yielding with special reference to the yield-point phenomena of iron and related bcc metals, Acta metallurgica 10 (1962) 727-738.

[17] S. Zhang, P. McCormick, Y. Estrin. The morphology of Portevin-Le Chatelier bands: finite element simulation for Al-Mg-Si, Acta materialia 49 (2001) 1087-1094.

[18] M. Lebyodkin, L. Dunin-Barkowskii, Y. Brechet, Y. Estrin, L. Kubin. Spatiotemporal dynamics of the Portevin-Le Chatelier effect: experiment and modelling, Acta Materialia 48 (2000) 2529-2541.

[19] G. Ananthakrishna. Current theoretical approaches to collective behavior of dislocations, Physics Reports 440 (2007) 113-259.

[20] M. Wagenhofer, M. Erickson-Natishan, R.W. Armstrong, F.J. Zerilli. Influences of strain rate and grain size on yield and serrated flow in commercial Al-Mg alloy 5086, Scripta Materialia 41 (1999) 1177-1184.

[21] C. Caceres, A. Rodriguez. Acoustic emission and deformation bands in Al$2.5 \% \mathrm{Mg}$ and $\mathrm{Cu}-30 \% \mathrm{Zn}$, Acta Metallurgica 35 (1987) 2851-2864.

[22] A. Ziegenbein, P. Hähner, H. Neuhäuser. Correlation of temporal instabilities and spatial localization during Portevin-LeChatelier deformation of $\mathrm{Cu}-10$ at.\% $\mathrm{Al}$ and $\mathrm{Cu}-15$ at.\% Al, Computational Materials Science 19 (2000) 27-34.

[23] Q. Zhang, Z. Jiang, H. Jiang, Z. Chen, X. Wu. On the propagation and pulsation of Portevin-Le Chatelier deformation bands: An experimental study with digital speckle pattern metrology, International Journal of Plasticity 21 (2005) 2150-2173.

[24] M.A. Sutton, J.J. Orteu, H. Schreier. Image correlation for shape, motion and deformation measurements: basic concepts, theory and applications, Springer Science \& Business Media, 2009.

[25] P.D. Zavattieri, V. Savic, L.G. Hector Jr, J.R. Fekete, W. Tong, Y. Xuan. Spatio-temporal characteristics of the Portevin-Le Châtelier effect in austenitic steel with twinning induced plasticity, International Journal of Plasticity 25 (2009) 2298-2330.

[26] H. Louche, A. Chrysochoos. Thermal and dissipative effects accompanying Lüders band propagation, Materials Science and Engineering: A 307 (2001) 15-22.

[27] H. Louche, P. Vacher, R. Arrieux. Thermal observations associated with the Portevin-Le Châtelier effect in an Al-Mg alloy, Materials Science and Engineering: A 404 (2005) 188-196. 
[28] N. Ranc, D. Wagner. Some aspects of Portevin-Le Chatelier plastic instabilities investigated by infrared pyrometry, Materials Science and Engineering: A 394 (2005) 87-95.

[29] S. Klein, M. Staring, K. Murphy, M.A. Viergever, J.P. Pluim. Elastix: a toolbox for intensity-based medical image registration, IEEE transactions on medical imaging 29 (2010) 196-205.

[30] C. Efstathiou, H. Sehitoglu. Strain hardening and heterogeneous deformation during twinning in Hadfield steel, Acta Materialia 58 (2010) 1479-1488.

[31] A. Chrysochoos, H. Louche. An infrared image processing to analyse the calorific effects accompanying strain localisation, International journal of engineering science 38 (2000) 1759-1788.

[32] A. Morabito, A. Chrysochoos, V. Dattoma, U. Galietti. Analysis of heat sources accompanying the fatigue of 2024 T3 aluminium alloys, International Journal of Fatigue 29 (2007) 977-984.

[33] M.R. Wenman, P.R. Chard-Tuckey. Modelling and experimental characterisation of the Lüders strain in complex loaded ferritic steel compact tension specimens, International Journal of Plasticity 26 (2010) 1013-1028.

[34] A. Rusinek, J.R. Klepaczko. Experiments on heat generated during plastic deformation and stored energy for TRIP steels, Materials \& Design 30 (2009) 35-48.

\section{Tables}

Table 1 Tensile properties of the medium Mn TRIP steel.

\begin{tabular}{rrcccc}
\hline $\begin{array}{r}\text { Upper } \\
\text { yield stress } \\
\sigma_{U}(\mathrm{MPa})\end{array}$ & $\begin{array}{c}\text { Lower } \\
\text { yield stress } \\
\sigma_{L}(\mathrm{MPa})\end{array}$ & $\begin{array}{c}\text { UTS } \\
(\mathrm{MPa})\end{array}$ & YPE & $\begin{array}{c}\text { Lüders } \\
\text { strain } \varepsilon_{L}\end{array}$ & $\begin{array}{c}\text { Elongat } \\
\text { ion to failure } \\
\varepsilon_{f}\end{array}$ \\
\hline 817 & 782 & 1012 & 0.0046 & 0.095 & 0.423 \\
\hline
\end{tabular}

Table 2 The estimated energy terms $Q_{p}$ and $Q_{\text {total }}$ in stages II and III.

\begin{tabular}{cccc}
\hline & $Q_{p}\left(\mathrm{~J} \mathrm{~m}^{-3}\right)$ & $Q_{\text {total }}\left(\mathrm{J} \mathrm{m}^{-3}\right)$ & $\delta$ \\
\hline Stage II & $3.9 \times 10^{7}$ & $6.1 \times 10^{7}$ & $56.4 \%$ \\
Stage III & $15.5 \times 10^{7}$ & $15.9 \times 10^{7}$ & $2.6 \%$ \\
\hline
\end{tabular}

\section{Figures Captions}

Fig. 1. EBSD phase map of the medium Mn TRIP steel. Austenite and ferrite are represented by blue and red color, respectively.

Fig. 2. Macroscopic engineering stress-strain curve of the specimen in the tensile test.

Fig. 3. (a) Evolution of the $1 \mathrm{D}$ axial strain $\bar{\varepsilon}_{y y}$ during the tensile test; (b) Evolution of the 1D incremental axial strain $\Delta \bar{\varepsilon}_{y y}$ during the tensile test. 
Fig. 4. (a) A succession of $2 \mathrm{D}$ axial strain $\left(\varepsilon_{y y}\right)$ fields at the selected moments during the Lüders band nucleation; (b) Evolution of mean local Lüders strain during the Lüders band nucleation.

Fig. 5. (a) A succession of $2 \mathrm{D}$ axial strain $\left(\varepsilon_{y y}\right)$ fields at the selected moments during the Lüders and propagation; (b) Local Lüders strain evolution of three selected points; (c) A succession of 2D axial strain $\left(\varepsilon_{y y}\right)$ fields at the selected moments during the propagation of the first PLC band; (d) Local PLC strain evolution of three selected points.

Fig. 6. Evolution of the average temperature increment $\overline{\bar{\theta}}$ and overall heat source $\overline{\bar{s}}$ versus engineering strain during the tensile test.

Fig. 7. (a) Evolution of the 1D temperature increment $\bar{\theta}$ during the tensile test; (b) Evolution of the 1D heat source $\bar{s}$ during the tensile test.

Fig. 8. Temperature and heat source evolution for a given pixel $(50,16)$.

Fig. 9. (a) The representative heat source fields of the eight principal PLC bands; (b) Axial profiles of the eight representative heat source fields along the midline of the specimen.

Fig. 10. (a) Estimated propagation velocity $V_{P L C}$ of the eight principal PLC bands versus axial strain; (b) Estimated time interval $\Delta T_{P L C}$ of the eight principal PLC bands versus axial strain.

Fig. 11. (a) Evolution of the volume fraction of austenite measured at the selected moments in a tensile test using XRD technique; (b) The distribution of HV along the loading direction of the specimen surface partially experienced by Lüders deformation in a tensile testing. 


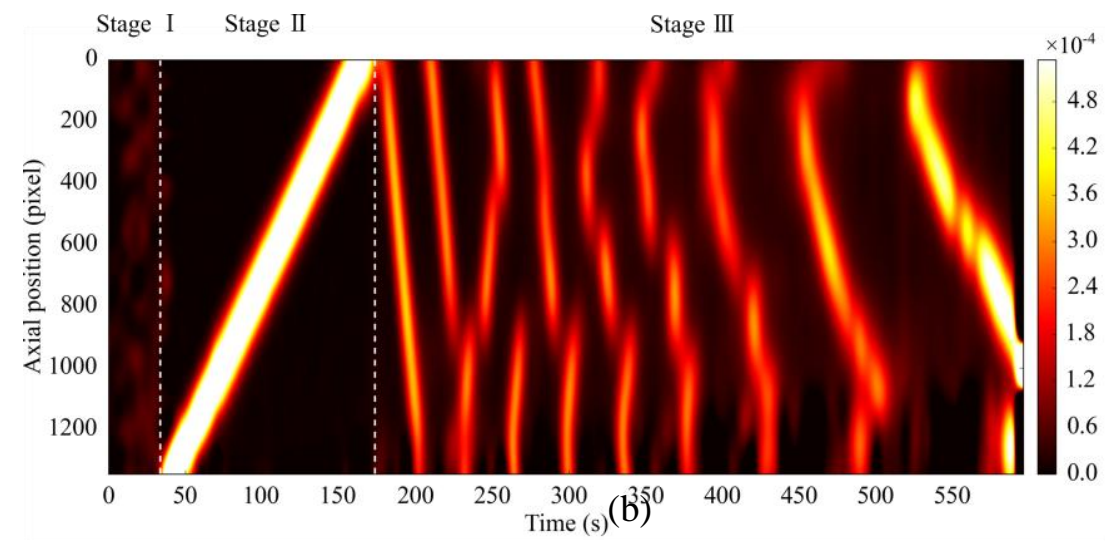

Fig. 3. (a) Evolution of the 1D axial strain $\bar{\varepsilon}_{y y}$ during the tensile test; (b) Evolution of the 1D incremental axial strain $\Delta \bar{\varepsilon}_{y y}$ during the tensile test.
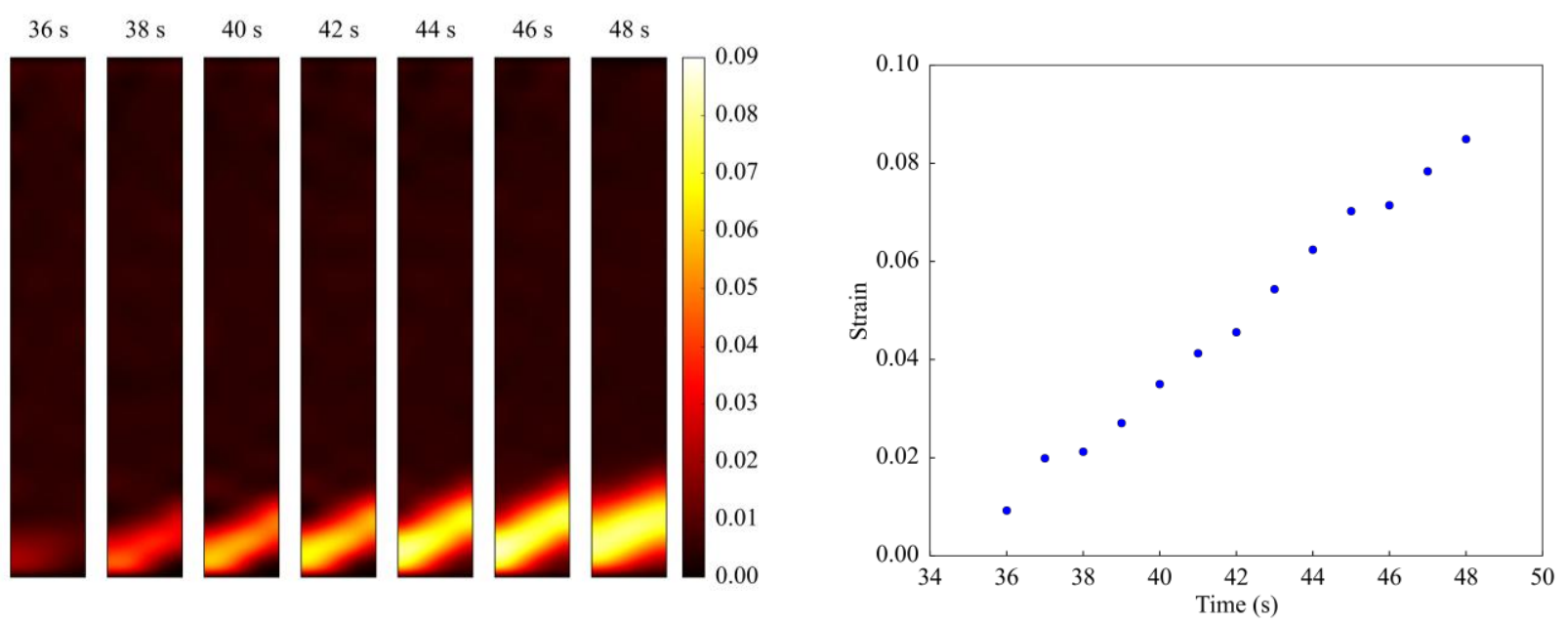

(a)

(b)

Fig. 4. (a) A succession of $2 \mathrm{D}$ axial strain $\left(\varepsilon_{y y}\right)$ fields at the selected moments during the Lüders band nucleation; (b) Evolution of mean local Lüders strain during the Lüders band nucleation. 

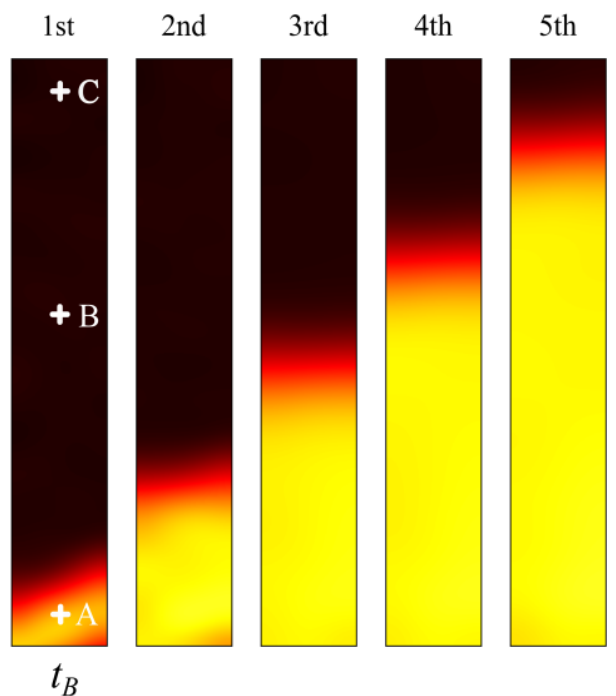

$t_{B}$
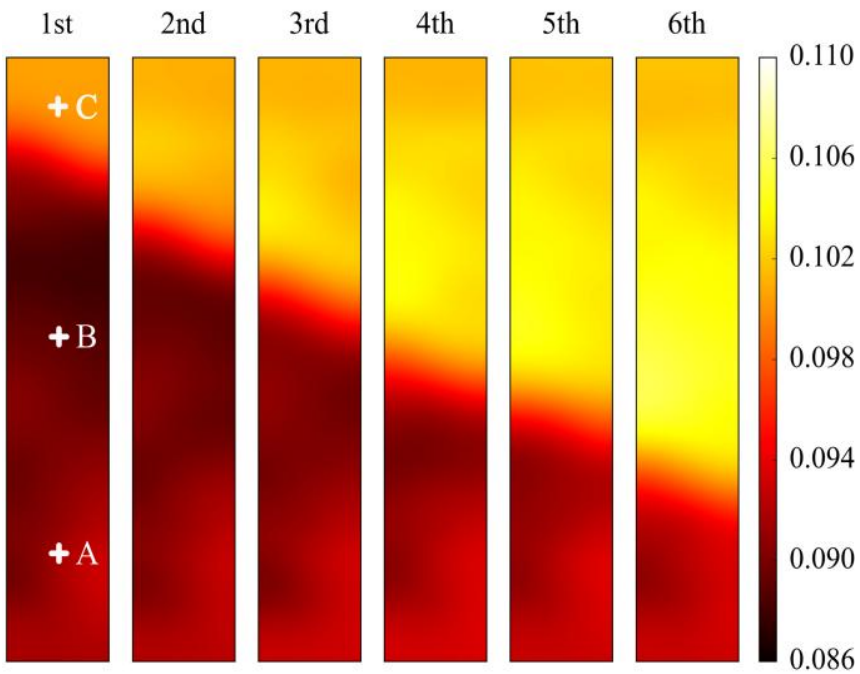

(c)

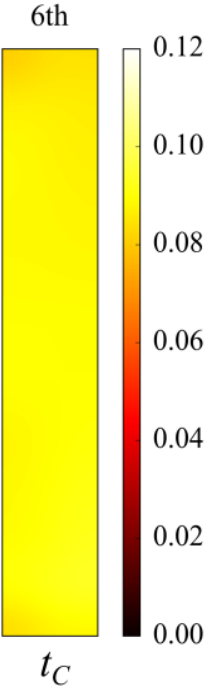

(a) (b)

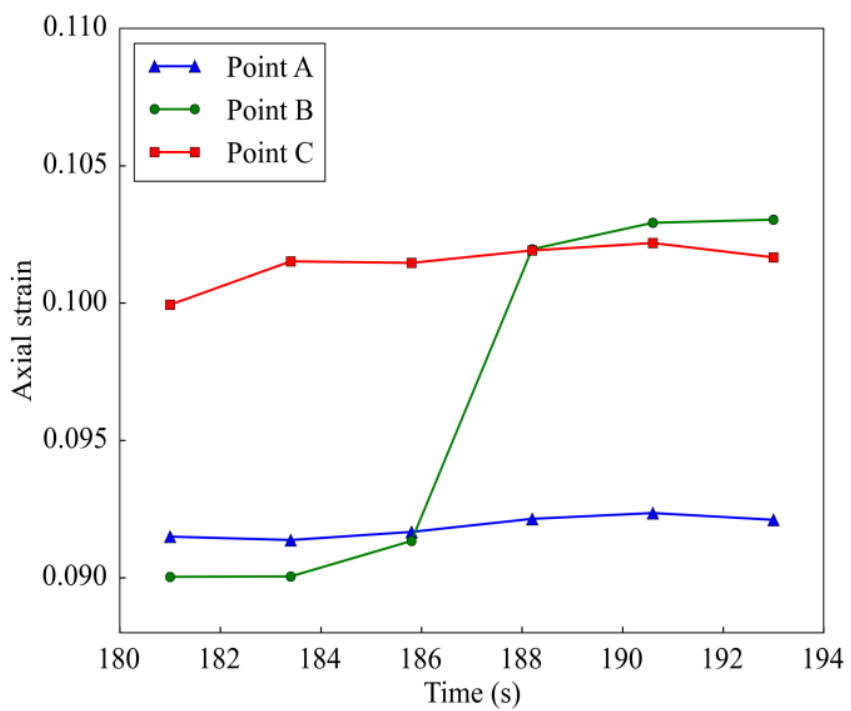

(d)

Fig. 5. (a) A succession of $2 \mathrm{D}$ axial strain $\left(\varepsilon_{y y}\right)$ fields at the selected moments during the Lüders and propagation; (b) Local Lüders strain evolution of three selected points; (c) A succession of $2 \mathrm{D}$ axial strain $\left(\varepsilon_{y y}\right)$ fields at the selected moments during the propagation of the first PLC band; (d) Local PLC strain evolution of three selected points. 


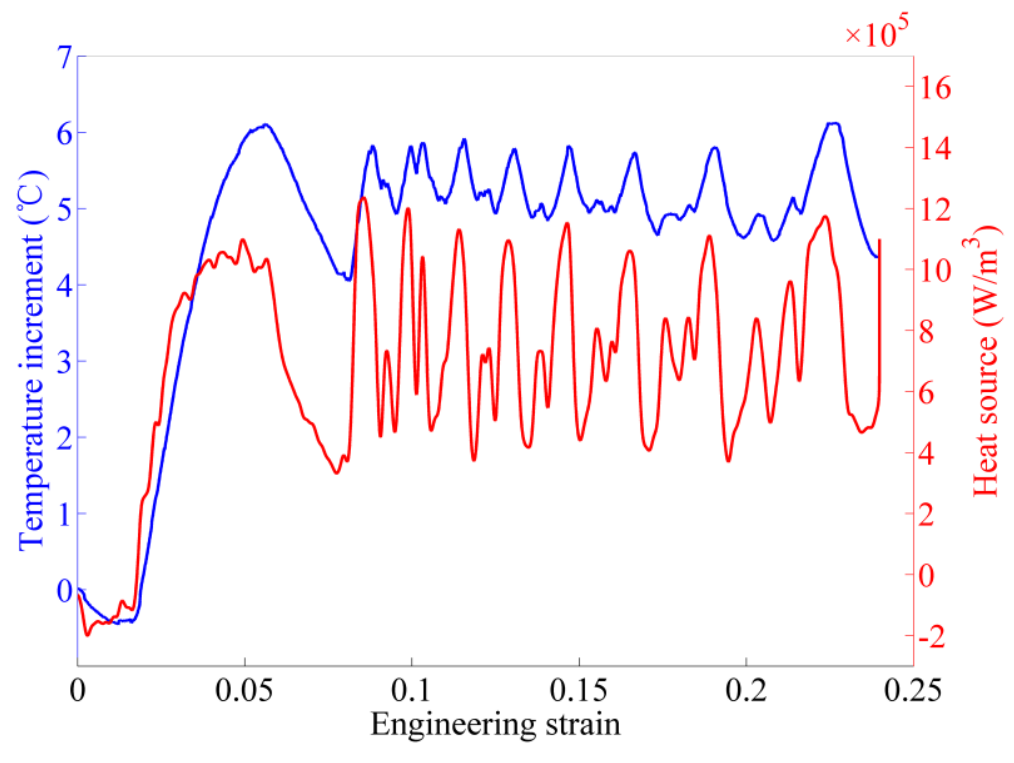

Fig. 6. Evolution of the average temperature increment $\overline{\bar{\theta}}$ and overall heat source $\overline{\bar{s}}$ versus engineering strain during the tensile test.

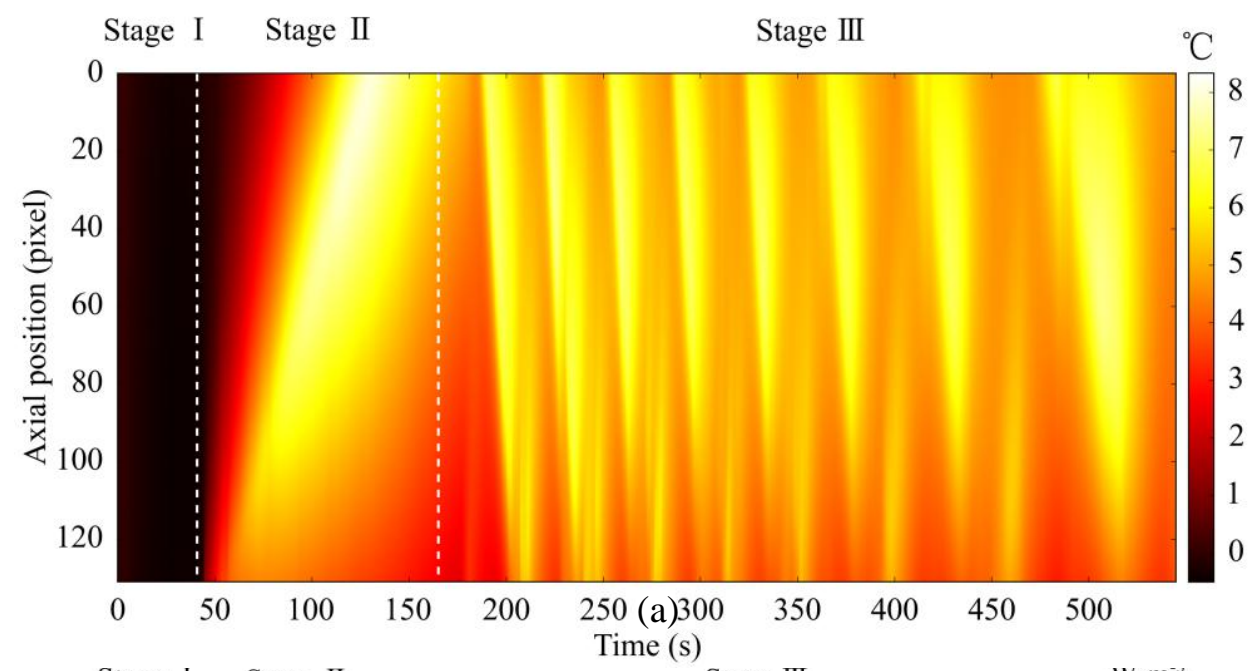

Fig.

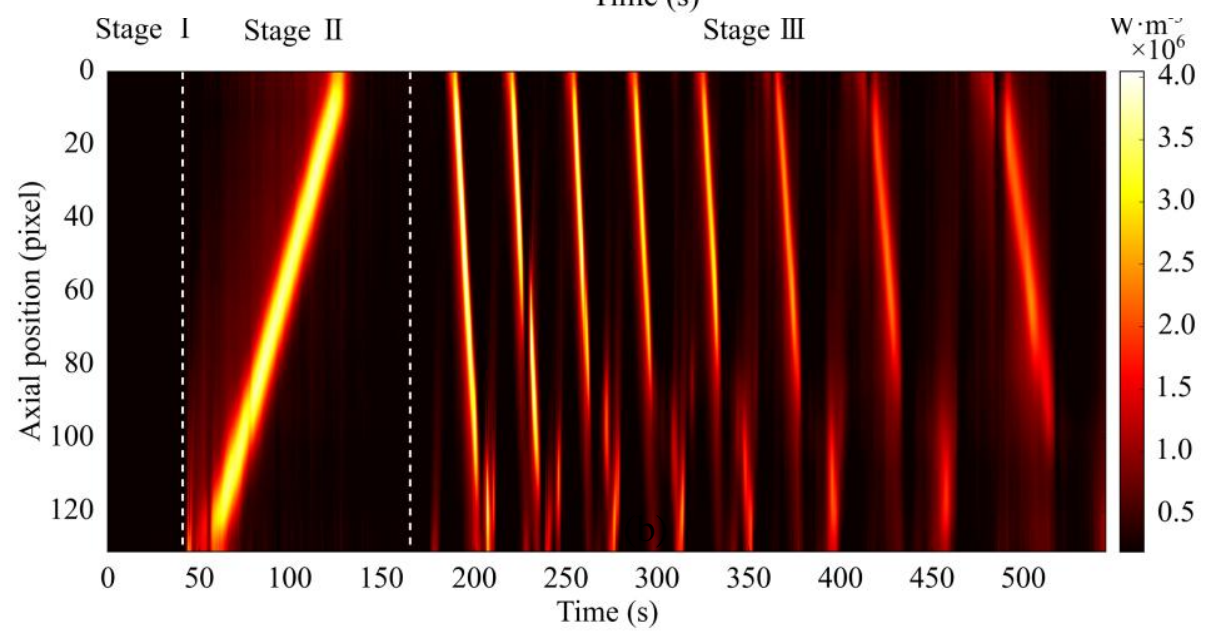

Evolution

of the 1D temperature increment $\bar{\theta}$ during the tensile test; (b) Evolution of the 1D heat source $\bar{s}$ during the tensile test. 


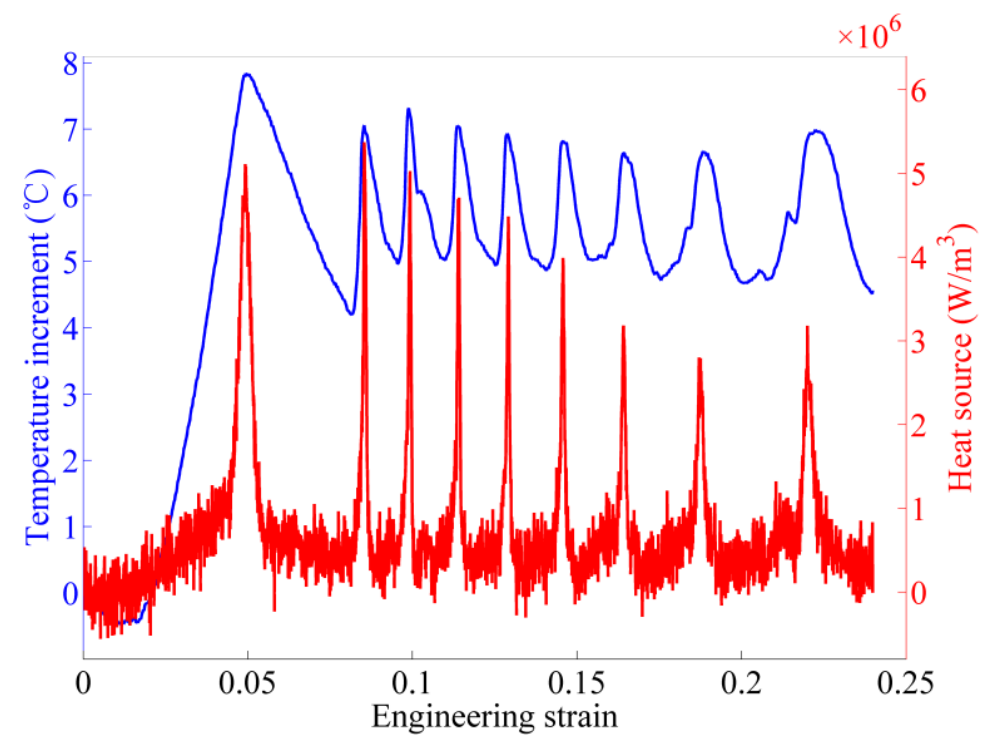

Fig. 8. Temperature and heat source evolution for a given pixel $(50,16)$.
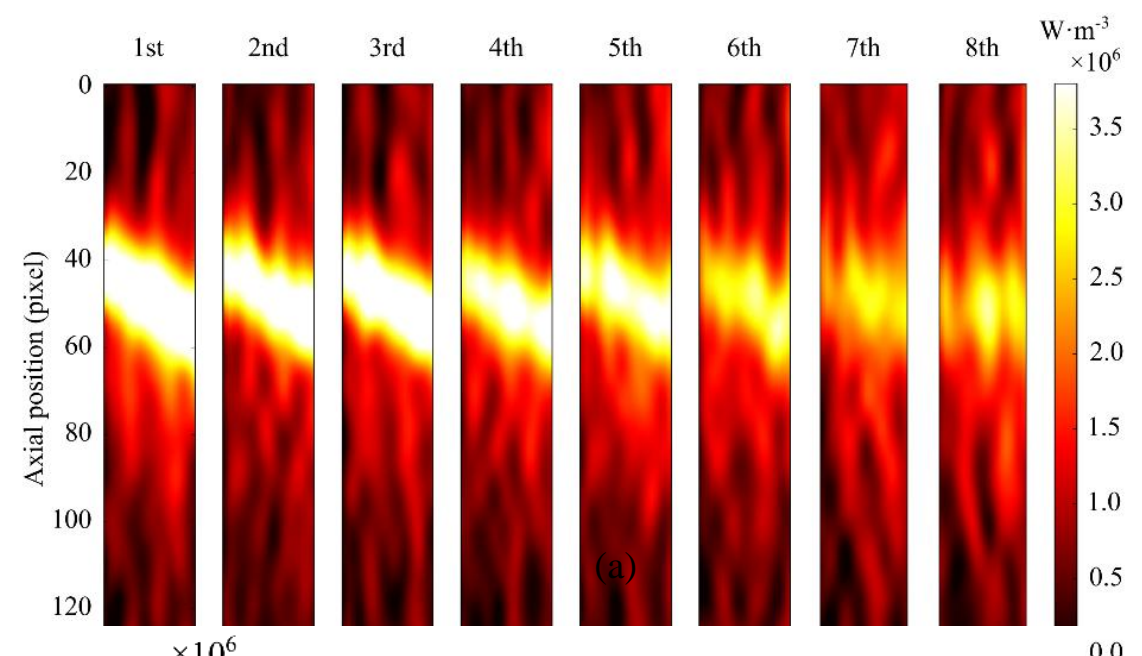

Fig. 9. (a) Th PLC bands; (b) Axic

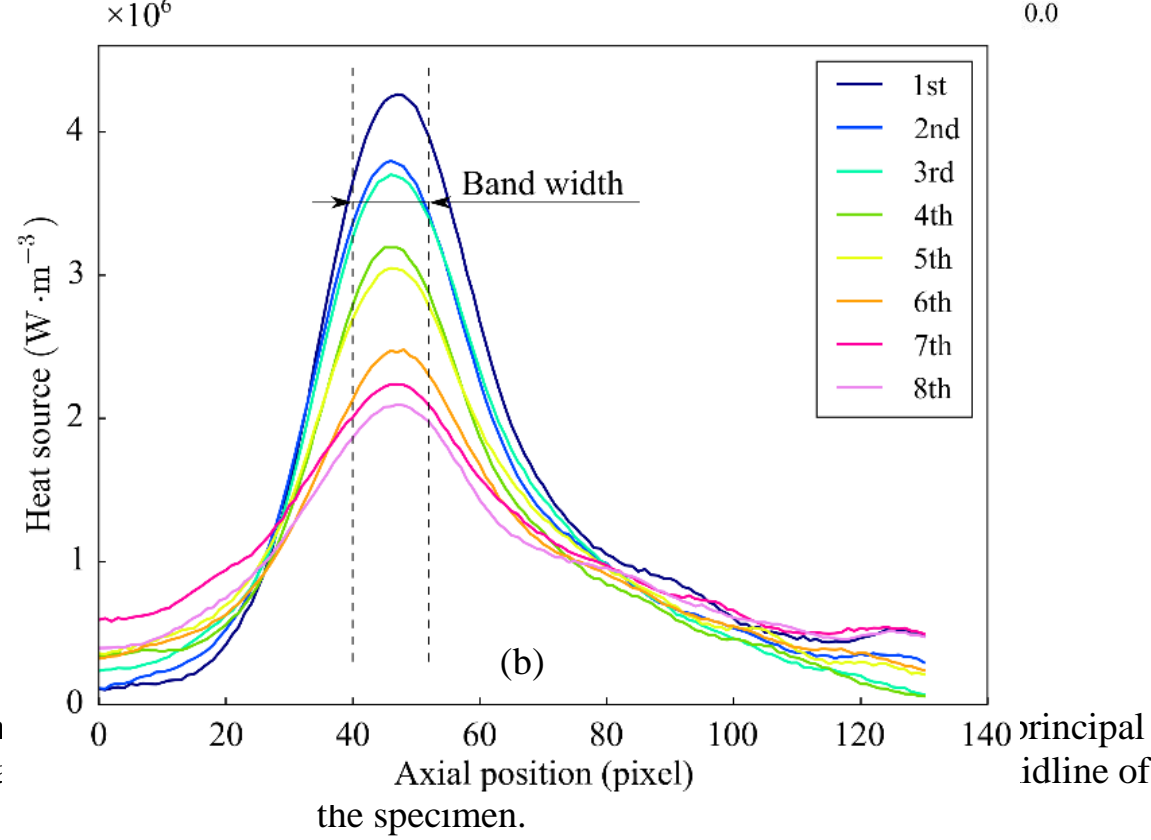



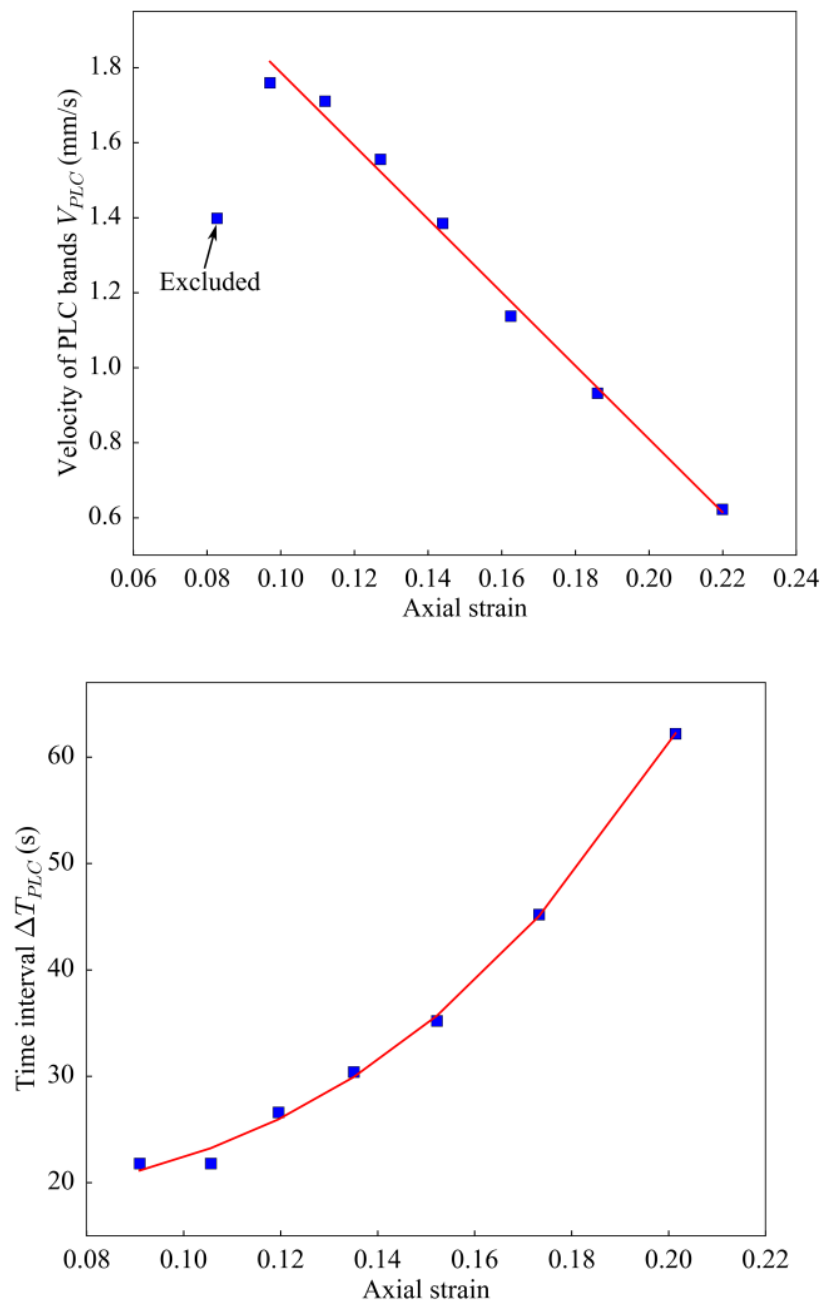

(a)

(b)

Fig. 10. (a) Estimated propagation velocity $V_{P L C}$ of the eight principal PLC bands versus axial strain; (b) Estimated time interval $\Delta T_{P L C}$ of the eight principal PLC bands versus axial strain. 


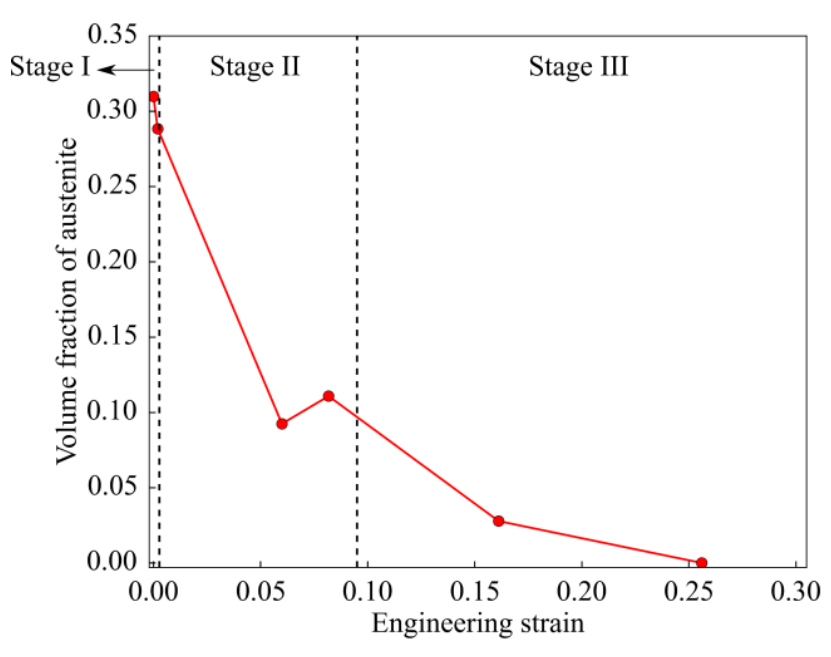

(a)

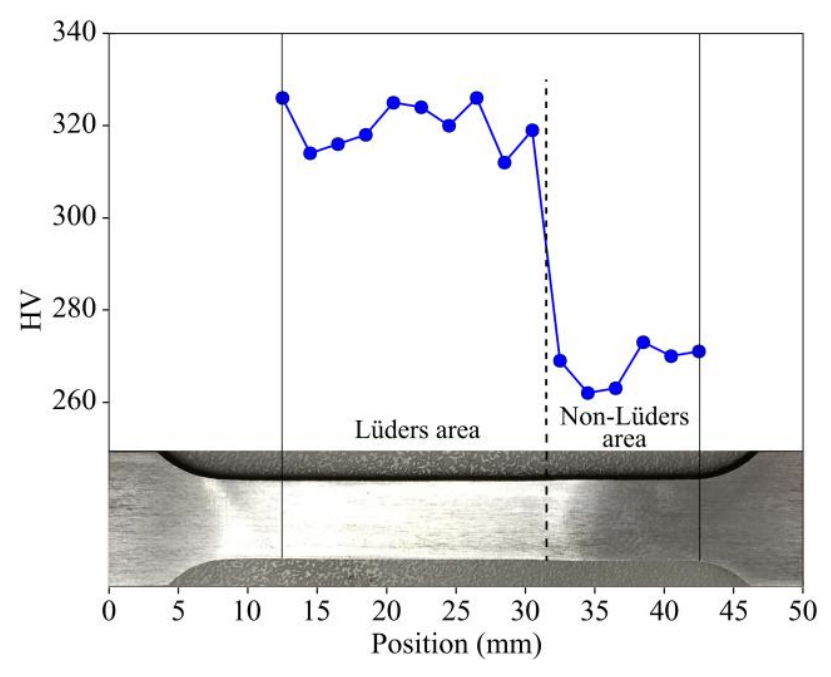

(b)

Fig. 11. (a) Evolution of the volume fraction of austenite measured at the selected moments in a tensile test using XRD technique; (b) The distribution of HV along the loading direction of the specimen surface partially experienced by Lüders deformation in a tensile testing. 


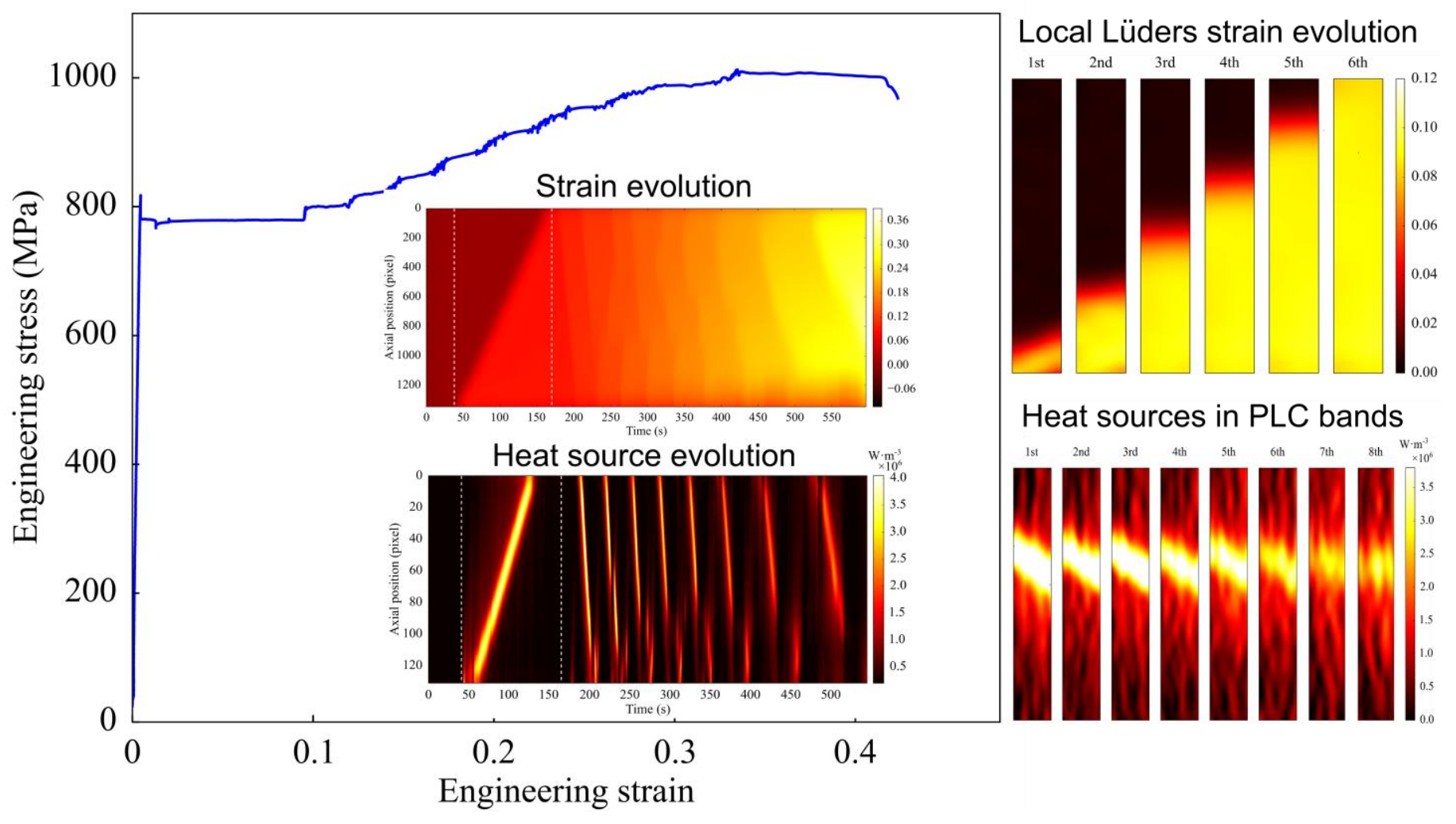

Kinematic and thermal characteristics of Lüders and Portevin-Le Châtelier bands in a medium Mn transformation-induced plasticity steel during tensile test. 